[0212-7199 (2004) 21: 11; pp 557-563] ANALES DE MEDICINA INTERNA Copyright (C) 2004 ARAN EDICIONES, S.L

AN. MED. InTERna (Madrid) Vol. 21, N. ${ }^{\circ} 11$, pp. 557-563, 2004

\title{
Importancia de la malnutrición y otros factores médicos en la evolución de los pacientes con fractura de cadera
}

\author{
M. GARCÍA LÁZARO, M. MONTERO PÉREZ-BARQUERO, P. CARPINTERO \\ BENÍTEZ ${ }^{1}$
}

Servicios de Medicina Interna I y de 'Traumatología. Hospital Provincial. Complejo Universitario Reina Sofía. Córdoba

THE ROLE OF MALNUTRITION AND OTHER MEDICAL FACTORS IN THE EVOLUTION OF PATIENTS WITH HIP FRACTURE

\section{RESUMEN}

Debido al progresivo envejecimiento de la población, la fractura de cadera es una patología cada vez más frecuente, que además conlleva una gran morbimortalidad y un enorme deterioro funcional. Habitualmente son pacientes ancianos, con un alto índice de comorbilidad y una marcada polimedicación. Es frecuente que al ingreso presenten desnutrición, sobre todo de predominio proteico, y que desarrollen complicaciones médicas intrahospitalarias. Todos estos factores, más que los meramente quirúrgicos, influyen de forma negativa en el pronóstico vital y funcional de estos enfermos. Por ello, sería necesario mejorar el control de la patología previa del paciente durante su hospitalización, valorar y tratar la malnutrición y prevenir las complicaciones médicas para intentar mejorar el pronóstico de estos enfermos. Para ello creemos que es necesario que sean atendidos por equipos multidisciplinarios y pensamos que el internista debería formar parte de estos equipos.

PALABRAS CLAVE: Fractura de cadera. Malnutrición. Evolución funcional. Mortalidad. Factores médicos.

\begin{abstract}
As the population progressively ages, hip fractures have become increasingly common and are associated with high morbidity and mortality and a pronounced decline in functional status. Hip fractures frequently occur in elderly patients with a high rate of comorbidity and polymedication. Patients hospitalised with hip fractures often display signs of protein malnutrition and may develop medical complications requiring intrahospital care. These factors, more than simply surgical ones, unfavourably influence the vital status and functional outcome of these patients. For this reason, it is necessary to improve the management of pre-existing conditions during hospitalisation, assess and treat malnutrition and prevent medical complications to achieve optimal outcomes for these patients. With this objective, we believe that care should be provided by multidisciplinary teams in close partnership with internists.
\end{abstract}

KEY WORDS: Hip fracture. Malnutrition. Functional outcome. Morbimortality. Medical factors.

García Lázaro M, Montero Pérez-Barquero M, Carpintero Benítez P. Importancia de la malnutrición y otros factores médicos en la evolución de los pacientes con fractura de cadera. An Med Interna (Madrid) 2004; 21: 557-563.

\section{INTRODUCCIÓN}

El número de ancianos crece de forma continua en todos los países desarrollados, alcanzando la población edades cada vez más elevadas. En nuestro país, las previsiones para el año 2010 son de 6.200.000 individuos con más de 65 años y, lo que es más importante, 1.500 .000 por encima de los 80 años. Se estima que en 2020 serán el 17,3\% de la población (1).

Las caídas y sus traumatismos relacionados son uno de los problemas más serios que sufren los ancianos. Se calcula que un $35-40 \%$ de las personas mayores de 65 años sufren una caí- da al año y por encima de los 75 años este porcentaje es aún mayor (2). Dentro de los traumatismos producidos por estas caídas destacan las fracturas de cadera por su frecuencia, su efecto devastador sobre la calidad de vida, su mortalidad y su enorme coste económico (3). Si se mantienen la tendencia demográfica actual, la incidencia, ya de por sí alta, de estas fracturas puede duplicarse o triplicarse a mediados del siglo XXI, lo que nos da una idea del importante problema de salud pública que suponen. El tratamiento correcto e integral de la fase aguda tiene una gran importancia en la evolución posterior del paciente y en él se consume una parte importante del coste de su asistencia.

Trabajo aceptado: 16 de julio de 2004 
Anualmente se producen en España unas 30.000 fracturas de cadera, incidencia similar a la de otros países del área mediterránea y menor que la de los países nórdicos. La sufren más las mujeres (un tercio de las ancianas mayores de 80 años sufre este tipo de fractura) con una relación que puede llegar a 3:1 ó 4:1 (4,5-10). La mayoría de los pacientes precisa tratamiento quirúrgico para intentar recuperar la capacidad de marcha prefractura. Esta recuperación, junto con la prevención de las complicaciones propias de la inmovilización prolongada (patología tromboembólica, úlceras por decúbito, deterioro de la función pulmonar, neumonías, etc.), mediante una movilización precoz postquirúrgica, constituyen los objetivos fundamentales del tratamiento (11).

\section{CARACTERÍSTICAS DE LOS PACIENTES QUE SUFREN UNA FRACTURA DE CADERA}

Como hemos comentado, estas fracturas ocurren sobre todo en edades tardías de la vida (con una media de edad de 80 años) $(4,6,7,12-15)$, por la mayor incidencia en estas edades de osteoporosis, patologías que predisponen a las caídas y agravan sus consecuencias, polimedicación y otros factores de riesgo (16).

Los pacientes suelen tener un alto índice de Charlson, con un número de enfermedades previas entre 3,3 y $4,35(9,17)$. Los varones suelen tener mayor comorbilidad $(18,19)$. Como consecuencia, existe una marcada polimedicación en estos pacientes, con una media en torno a 2,5-3,5 fármacos distintos/día $(9,20)$, lo que dificulta su manejo por parte de los equipos quirúrgicos. La toma de medicación con efecto sedante es muy frecuente, con porcentajes que se acercan al 40\% (20-22).

$\mathrm{Al}$ ingreso, un alto porcentaje de pacientes presentan cifras bajas de hemoglobina (23), por diversas causas: los ancianos sufren con frecuencia patología crónica que se acompaña de anemia de patrón inflamatorio crónico, pero también es frecuente la anemia ferropénica. A esto se añade la pérdida sanguínea en el foco de fractura. Asimismo está descrita una alta proporción de linfopenia $(8,24-26)$, grados variables de deshidratación y una marcada incidencia de déficit de vitamina D $(27,28)$. En nuestro país, se ha comunicado un déficit de vitamina D hasta en el $91 \%$ de ancianos con fractura de cadera (29). Estos datos subrayan el papel que tiene este déficit como factor de riesgo, y aunque ciertamente no es el más importante, sí es el más fácil de corregir.

Las complicaciones intrahospitalarias son muy frecuentes, habiéndose comunicado incidencias de hasta el $84 \%$ de los enfermos $(9,30)$.

La mortalidad intrahospitalaria de las series publicadas oscila entre el 4,76 y el 16,9\% (17,24,31-33). En nuestro país varía entre un 4,2 y un $8 \%(4,6,9,19,34,35)$. A los 3 meses de la fractura, la mortalidad oscila entre un 8 y un $27 \%(25,36-$ 38). Al año la mortalidad varía entre el 20,5 y el $31,6 \%$ $(18,19,24,25,35,37-40)$. Tras este periodo de 10-12 meses, el riesgo de mortalidad se iguala con las personas de similar edad y sexo que no se han fracturado la cadera.

Los pacientes que no fallecen sufren un deterioro funcional importante. La valoración de la recuperación funcional es especialmente dificultosa, debido a la falta de estandarización de los niveles funcionales $(11,41)$. En algunas series se emplea como indicador de recuperación funcional las actividades de la vida diaria (42), siendo la evolución funcional en estas actividades paralela a la recuperación de la autonomía en la marcha (39). En general, en pacientes de cualquier edad, el $40-50 \%$ recupera su nivel funcional previo y el 20-30\% quedan encamados permanentemente $(8,39,43,44)$, aunque hay series con mejores resultados (6).

\section{IMPORTANCIA DEL ESTADO NUTRICIONAL EN LA EVOLUCIÓN} DE LOS PACIENTES CON FRACTURA DE CADERA

La nutrición tiene una gran influencia sobre la salud del individuo y sobre la expectativa de vida. Paralelamente con el envejecimiento poblacional, los problemas nutricionales de la vejez han ido cobrando protagonismo, pues la desnutrición condiciona una mayor morbimortalidad general. El envejecimiento conlleva toda una serie de cambios que tienen una repercusión directa sobre el estado nutricional (alteraciones sensoriales, bucales o digestivas, menor autonomía funcional, demencia, viudedad, efectos adversos medicamentosos, etc.), haciendo de los ancianos una población especialmente susceptible de padecer problemas nutricionales.

Recordemos que la desnutrición comienza cuando el aporte de nutrientes es insuficiente para cubrir las necesidades del paciente, y progresa hasta producir alteraciones funcionales y anatómicas, caracterizadas por una pérdida de grasa corporal y/o masa libre de grasa y esto permite distinguir dos tipos clásicos de malnutrición (45):

- El marasmo o caquexia del adulto, debido a una pobre ingesta calórica, con ingesta proteica baja, pero proporcional a la de calorías. La grasa y el músculo esquelético son movilizados como fuente de energía, disminuyendo el peso y las medidas antropométricas. Las proteínas viscerales suelen mantenerse normales y también se conserva el estado inmunitario.

-El kwasiorkor, consecuencia de un inadecuado aporte proteico, aunque el aporte calórico sea normal. Los pacientes conservan las medidas antropométricas y a menudo parecen bien nutridos, pero existe una importante depleción de proteínas viscerales, una alteración de la respuesta inmune y puede haber edemas generalizados.

La desnutrición calórico-proteica o mixta es el tipo más frecuente, pues difícilmente hallaremos en nuestro medio un tipo puro de desnutrición, si bien puede haber predominio de uno u otro tipo dentro de una desnutrición mixta. Las alteraciones funcionales son, no obstante, las más importantes, ya que originan la mayoría de las complicaciones relacionadas con el déficit nutricional, como son la menor resistencia a las infecciones, el retraso en la curación de las heridas o las dificultades para toser o moverse (46).

Aparte de la insuficiente ingesta calórica y/o proteica, se pueden ver afectados otros nutrientes, como el hierro, el calcio y la vitamina $\mathrm{D}$, que puede favorecer la osteoporosis grave. La malnutrición aumenta además el riesgo de fracturas osteoporóticas a través otros mecanismos: por un lado, una ingesta proteica por debajo de las necesidades compromete no solo al pico de masa ósea alcanzado, sino también a su preservación a lo largo de los años (47); por otro lado, la malnutrición aumenta la propensión a las caídas debido a la debilidad muscular y al empeoramiento de la coordinación motora y por último, se produce una falta de protección de los tejidos blandos alrededor de las caderas (48).

La malnutrición, que es frecuente entre la población anciana, está aún más presente entre los ancianos hospitalizados 
por cualquier causa, aunque es muy variable de un estudio a otro, con cifras que van del 22 al 70\% (49,50). Esteban Pérez encuentra entre los ancianos hospitalizados en nuestro país un $50,5 \%$ de desnutridos (51). Numerosos autores describen también una alta prevalencia de malnutrición entre los enfermos con fractura de cadera, tanto en Europa como en USA o Nueva Zelanda. Las cifras oscilan entre un 31 y un $88 \%$ en función de los criterios empleados $(7,8,14,24,25,52-59)$. Los porcentajes menores coinciden con la utilización de criterios antropométricos, los mayores con la de criterios analíticos, y los intermedios con la combinación de ambos. La mayor frecuencia de diagnóstico de malnutrición cuando se usan criterios analíticos puede deberse a que albúmina, prealbúmina y transferrina pueden comportarse también como reactantes negativos de fase aguda (60). El problema que supone la falta de criterios estandarizados para el diagnóstico de malnutrición se pone de relieve con el trabajo de Joosten y cols. (61) donde al evaluar 151 ancianos ingresados por patología aguda médica, aplicando 6 sistemas diagnósticos diferentes de 6 estudios previos, hallaron una proporción de pacientes malnutridos que variaba entre 6,5 y 85\%, según el sistema empleado. Esto dificulta enormemente la comparación de resultados y la decisión de tratar. No obstante, a pesar de estas dificultades, la negativa influencia de la malnutrición en la morbimortalidad postfractura debe estimularnos a no olvidar el estudio nutricional dentro de la valoración global del paciente.

En este sentido, diversos autores han comunicado una hospitalización más larga, reflejo de una peor evolución, entre los pacientes con malnutrición proteica $(8,55,62)$ o calórica $(62)$. Schurch, Tkatch, y Delmi y cols. observan también una menor estancia media en tres estudios randomizados, en el grupo de pacientes con fractura de cadera que recibió suplementos dietéticos proteicos $(32,33,63)$.

Por otra parte, hay estudios que constatan que los pacientes con malnutrición calórico-proteica sometidos a intervenciones quirúrgicas presentan una mayor tasa de complicaciones totales (24), tasa que disminuye si los pacientes reciben suplementos proteicos $(32,33)$. Asimismo, se ha descrito un aumento de complicaciones infecciosas en los pacientes malnutridos (64-66), lo cual no es de extrañar si tenemos en cuenta que la malnutrición tiene un efecto deletéreo sobre la funcionalidad de los linfocitos, además de aumentar el porcentaje de células nulas y disminuir las células T-helper $(67,68)$.

Por otro lado, la malnutrición proteica sobre todo, pero también la calórica, predisponen al desarrollo de las temidas escaras (69-72), lo que indica que hay que vigilar estrechamente el estado nutricional de los pacientes con fractura de cadera, introduciendo soporte nutricional en aquellos enfermos con alto riesgo, tanto para intentar evitar la aparición de escaras, como para favorecer su curación.

En cuanto a la mortalidad, diversos autores constatan que un estado nutricional deficitario, sobre todo de predominio proteico $(8,11,24,25)$ pero también calórico o mixto $(17,31-33,62)$ aumenta la mortalidad precoz y tardía de estos pacientes. Apoyando esta hipótesis, varios trabajos han encontrado una menor mortalidad aguda en pacientes con fractura de cadera a los que se administra suplementos nutricionales vía oral (33) o vía enteral (23) y se ha visto un efecto positivo de estos suplementos vía enteral incluso en la mortalidad a 6 meses (73).

En ancianos ingresados por patología médica con malnutrición proteica (74) o mixta (49) también se ha descrito una mayor mortalidad hospitalaria. Los niveles bajos de albúmina, colesterol, hematocrito, hemoglobina, IMC y pliegue tricipital aumentan el riesgo de mortalidad en ancianos institucionalizados (75) y en este grupo poblacional, un valor por debajo del $5^{\circ}$ percentil del pliegue tricipital se ha asociado a un incremento del riesgo relativo de morir por cualquier causa (76).

Por otro lado, la malnutrición calórico-proteica es claramente un factor de mal pronóstico funcional $(24,33)$ y conlleva un peor grado de rehabilitación (52). Esto también ocurre en pacientes no quirúrgicos (77). El bajo peso y la circunferencia muscular del brazo se asocian a fatiga muscular (78) y así como la debilidad muscular que produce la malnutrición calórico-proteica favorece las caídas (48), también dificulta la recuperación. Confirma la importancia de la malnutrición en el pronóstico funcional de los pacientes con fractura de cadera el trabajo de Bastow y cols., donde la recuperación funcional es más rápida en el subgrupo de su serie con mejores valores de peso, pliegue tricipital y circunferencia del brazo y también en el subgrupo con peores valores que recibieron suplementos nutricionales vía enteral (23). En la misma línea, Schurch y cols. describen también una recuperación funcional más rápida y un aumento de la fuerza muscular del bíceps en los pacientes que recibieron suplementos nutricionales proteicos (63). En pacientes geriátricos malnutridos con patología médica también se ha descrito una mejoría funcional cuando reciben suplementos nutricionales $(79,80)$.

Todos estos datos nos deben estimular a incluir siempre el estudio nutricional, usando todas las variables posibles, en la valoración clínica de los ancianos con fractura de cadera, y a tenerlo en cuenta como marcador de mal pronóstico si los datos son anómalos.

OTROS FACTORES QUE INFLUYEN EN LA MORTALIDAD TRAS LA FRACTURA DE CADERA

Los factores que se asocian en los distintos estudios a una mayor mortalidad, se pueden ver resumidos en la tabla I.

TABLA I

FACTORES ASOCIADOS A MAYOR MORTALIDAD TRAS UNA FRACTURA DE CADERA

\begin{tabular}{ll}
\hline Asociación clara & $\begin{array}{l}\text { Resultados contradictorios } \\
\text { o ausencia de asociación }\end{array}$ \\
\hline Malnutrición & Tipo de fractura \\
Edad avanzada & Demora quirúrgica \\
Género masculino & \\
Mal nivel funcional previo & \\
Institucionalización previa & \\
Mayor comorbilidad & \\
Existencia de complicaciones médicas & \\
Mal nivel funcional al alta & \\
\hline
\end{tabular}

-Edad: la mayoría de los autores han constatado una mayor mortalidad tanto precoz $(4,11,17,36,37,43,81)$ como al año $(21,24,37,38,40)$ en los enfermos de más edad. Los pacientes mayores habitualmente son más frágiles, con una salud más precaria y peor respuesta ante las complicaciones, lo que conduce a una mayor mortalidad. Algunos autores han 
descrito efectivamente que, si no hay patología previa aparte de la fractura (19), o si son pacientes menores de 90 años sanos y sin complicaciones (31), la edad no condiciona la mortalidad. Más que la edad real, puede ser el estado de salud y la llamada edad biológica lo que marque el pronóstico, al menos hasta los muy ancianos.

-Género: son muchos los estudios donde se describe una mayor mortalidad tanto precoz $(4,6,11,20,36,37,82,83)$ como al año $(19,21,37,38,40)$ en los varones con fractura de cadera. Dado que la mortalidad se relaciona con el estado de salud prefractura, la mayor mortalidad de los varones parece ser reflejo de una salud más precaria con respecto a la mujer $(11,18,83)$.

-Nivel funcional previo: un peor estado funcional previo conlleva una mayor mortalidad precoz $(6,19,83)$ y a los 6 y 12 meses (19). En parte de los pacientes, el bajo nivel funcional se debe a un precario estado de salud y eso explicaría el mal pronóstico asociado a este factor, como refleja el trabajo de Mullen y cols. (31), donde el nivel funcional prefractura aumenta la mortalidad sólo en un subgrupo de enfermos con determinada patología previa, pero no si estaban sanos.

- La institucionalización previa del paciente conlleva mayor riesgo de mortalidad (37).

-Tipo de fractura: aunque algunos autores han descrito una mayor mortalidad precoz y tardía en las fracturas pertrocantéreas (37), la mayoría no encuentran mayor mortalidad en uno u otro tipo de fractura $(6,18,19,40,81)$.

-Demora quirúrgica: Los resultados publicados en la literatura son contradictorios. En algunos estudios la mortalidad precoz es menor en los pacientes operados en las primeras 24 horas (84), si bien habitualmente en el grupo en el que se retrasa la intervención hay un porcentaje significativamente mayor de pacientes con peor situación clínica, siendo ésta generalmente la causa de tal retraso (18), lo que puede incidir en la mayor mortalidad asociada a la demora quirúrgica descrita por algunos autores $(18,85)$. En otras series no se encuentra relación entre demora quirúrgica y la mortalidad precoz, a los 6 y a los 12 meses $(19,24,86,87)$. Mullen y cols. han descrito incluso una mayor mortalidad en los pacientes a los que se intervino en las primeras 24 horas, menor con un retraso de 24-72 horas y aún menor si se demoraba más de 72 horas, si bien consideraban solo los casos con algún tipo de patología médica aguda (31). Kenzora y cols. (88), incluso introduciendo una corrección según enfermedades asociadas (presencia de 4 o más), encuentra mayor mortalidad al año en los pacientes intervenidos en las primeras 24 horas que si se operan en 2-5 días ( $28 \%$ vs $4 \%$ ). La clave no parece ser los días transcurridos entre fractura y cirugía, sino que se espere lo suficiente en cada caso como para estabilizar correctamente la situación hidroelectrolítica y los demás problemas médicos del paciente y que éste llegue al quirófano en las mejores condiciones clínicas posibles $(11,89)$.

-Comorbilidad: Se ha descrito una mayor mortalidad precoz y tardía en pacientes con mayor comorbilidad en general $(11,19,37,83,88)$, con patología previa cerebrovascular o cardiaca $(19,82,86)$, EPOC o insuficiencia renal $(40,86)$, y estado mental previo precario $(6,19,40)$. El estudio de White y cols. (18), con una mortalidad al año de los pacientes con fractura de cadera con bajo riesgo según el sistema ASA (Sociedad Americana de Anestesistas) similar a la población general, corrobora la clara influencia de la comorbilidad en la mortalidad de estos pacientes. Es importante, por tanto, el especial cuidado de los pacientes con enfermedades asociadas y su adecuado control durante su hospitalización.

-Complicaciones: Las complicaciones médicas intrahospitalarias se asocian a una mayor mortalidad precoz y tardía $(17,19,31,81,82,88)$ y, por tanto, hay que prestar especial atención a su prevención y tratamiento.

-Nivel funcional al alta: La buena movilidad conseguida tras una fractura de cadera es un factor predictor positivo en cuanto a la mortalidad (90). Por otro lado se ha descrito una mayor mortalidad precoz y tardía en pacientes encamados al alta (31), pero considerado sólo aquellos casos en los que este estado se debía al precario estado de salud y no a indicación del cirujano de no cargar por fractura inestable.

\section{OTROS FACTORES QUE INFLUYEN EN LA EVOLUCIÓN} FUNCIONAL

Se pueden ver resumidos en la tabla II.

$$
\text { TABLA \| }
$$

FACTORES ASOCIADOS A PEOR EVOLUCIÓN FUNCIONAL TRAS UNA FRACTURA DE CADERA

\begin{tabular}{ll}
\hline Asociación clara & $\begin{array}{l}\text { Resultados contradictorios } \\
\text { o ausencia de asociación }\end{array}$ \\
\hline Malnutrición & Cénero \\
Edad avanzada & Tipo de fractura \\
Mal nivel funcional previo & Tipo de cirugía \\
Consumo excesivo de alcohol & Demora quirúrgica
\end{tabular}

Estancia media

Mayor comorbilidad

Existencia de complicaciones médicas

-Edad: La mayor edad predispone a una peor recuperación funcional general (42) y de la capacidad de marcha en las primeras semanas postfractura $(91,92)$, a los 4 y 6 meses $(93,94)$, y al año $(11,12,43,44,93,95)$. Parece claro que la edad del paciente es importante a lo largo de toda la evolución funcional (96). Los pacientes más ancianos suelen ser menos capaces de llevar a cabo el esfuerzo físico que requiere la recuperación de la capacidad de marcha.

-Género: Aunque algunos autores señalan una peor recuperación funcional al año de las mujeres $(11,12)$, en la mayoría de las series el sexo no influyó en la recuperación a los 3, 6 o 12 meses $(43,94,96,97)$.

- Estancia media y demora quirúrgica. La mayor estancia media, reflejo de una peor evolución hospitalaria, se asocia a una peor recuperación funcional $(12,44)$. Sobre la demora quirúrgica hay datos contradictorios en la literatura; algunos autores señalan que una demora quirúrgica mayor de 48 horas se asocia a peor evolución funcional $(91,98)$, mientras otros no corroboran estos datos $(12,95)$.

-Tipo de fractura: Su relación con la recuperación funcional no está clara. Algunos autores señalan una peor recuperación en las fracturas extracapsulares $(42,87,99)$, mientras otros no encuentran diferencias entre fracturas intra o extracapsulares $(12,97)$. Es posible que las fracturas extracapsulares tiendan a tener peor evolución funcional, por aparecer en edades más avanzadas que las intracapsulares (100) y por tanto en una población más frágil en todos los sentidos. 
-Tipo de cirugía: En general en los trabajos publicados no se encuentran diferencias en el resultado funcional a corto y a largo plazo entre la fijación interna y las prótesis $(42,98)$.

-Nivel funcional prefractura: A pesar de que los pacientes con mejor nivel funcional prefractura tienen mayor recorrido funcional que recuperar, y es más difícil recuperar un buen nivel prefractura que un nivel bajo $(11,44,95,97)$, al año estos pacientes consiguen mejores resultados funcionales y se recuperan mejor $(92,94,101)$.

-Hábitos tóxicos: El consumo excesivo de alcohol dificulta la recuperación funcional, debido en parte al deterioro cognitivo que produce, a la frecuente malnutrición de estos pacientes y a la toxicidad muscular del alcohol (102).

-Comorbilidad: En la mayoría de las series la comorbilidad predispone a una peor recuperación funcional a lo largo del primer año $(93,94,96,97)$. Los pacientes con mayor comorbilidad son más propensos a las complicaciones y su peor estado de salud perjudica su evolución funcional. Entre las patologías asociadas destaca la demencia como factor asociado a peor recuperación funcional a lo largo del primer año $(11,12,42,92,94,101,103)$. Si bien es cierto que el deterioro cognitivo retrasa o impide la necesaria colaboración del paciente para levantarse y caminar, esto no debe conducir a una actitud derrotista ante estos enfermos, pues se ha visto que con programas especiales intensivos de rehabilitación, pueden conseguir un buen resultado funcional (104). Los pacientes con ictus previo, al contrario de lo que se pudiera pensar, no recuperan peor su nivel funcional previo. En una larga serie de 862 pacientes no institucionalizados, Youm y cols. no encuentran una peor recuperación del nivel prefractura al año en este tipo de pacientes (105).

\section{Bibliografía}

1. Instituto Nacional de Estadística. Proyección de la población española para el periodo 1980-2010. Madrid, INE, 1989.

2. American Geriatrics Society, British Geriatrics Society, And American Academy of Orthopaedic Surgeons Panel on Falls Prevention. Guideline for the prevention of falls in older persons. Am Geriatr Soc 2001; 49: 664-72.

3. Barret J, Connor E. The economic and human cost of osteoporotic fracture. Am J Med 1995; 98: 35-75.

4. Serra JA, Garrido G, Vidán M, Marañón E, Brañas F, Ortiz J. Epidemiología de la fractura de cadera en ancianos en España. An Med Interna (Madrid) 2002; 19: 389-95.

5. Zuckerman JD, Skovron ML, Koval KJ, Aharonoff G, Frankel VH. Postoperative complications and mortality associated with operative delay in older patients who have a fracture of the hip. J Bone Joint Surg Am 1995; 77: 1551-6.

6. Pagès E, Cuxart A, Iborra J, Olona M, Bermejo B. Fracturas de cadera en el anciano: determinantes de mortalidad y capacidad de marcha. Med Clin (Barc) 1998; 110: 687-91.

7. Hanger HC, Smart EJ, Merrilees MJ, Frampton CM. The prevalence of malnutrition in elderly hip fracture patients. NZ Med J 1999; 112: 88-90.

8. Koval K, Maurer SG, Su ET, Aharonoff GB, Zuckerman JD. The effects of nutritional status on outcome after hip fracture. J Orthop Trauma 1999; 13: 164-9.

9. González-Montalbo JI, Alarcón T, Sáez P, Bárcena A, Gotor P, del Río M. La intervención geriátrica puede mejorar el curso clínico de los ancianos frágiles con fractura de cadera. Med Clin (Barc) 2001; 116: 1-5.

10. March LM, Cameron ID, Cumminf RG, Chamberlain AC, Schwarz JM, Brnabic AJ, et al. Mortality and morbidity after hip fracture: can evidence based clinical pathways make a difference? J Rheumatol 2000; 27: 2227-31.
-Existencia de complicaciones: Las complicaciones generales e infecciosas empeoraran la recuperación funcional de estos pacientes $(39,93)$. Es fundamental prevenir y tratar precozmente estas complicaciones para intentar mejorar el pronóstico funcional tras la fractura. Entre las complicaciones destacan los cuadros confusionales $(11,12,98)$. Estos cuadros, más frecuentes en pacientes de más de 80 años, con deterioro cognitivo previo o con marcada comorbilidad, se asocian a una peor recuperación funcional al mes, incluso de forma independiente a estos factores (106). Además, la mayoría de los pacientes que desarrollan cuadros confusionales tienen algún síntoma persistente hasta 6 meses después del alta y esto interfiere con las actividades de rehabilitación (107).

\section{CONCLUSIÓN}

Debemos destacar el efecto deletéreo de la malnutrición en la morbimortalidad y la evolución funcional tras la fractura de cadera. A pesar de esto, con frecuencia es un factor al que no se le presta la debida atención. Por otro lado, hay un predominio de factores puramente médicos que ejercen una marcada influencia en el pronóstico vital y funcional de este tipo de pacientes. Sin embargo, en muchos centros hospitalarios son atendidos por equipos exclusivamente quirúrgicos. Sería preciso estimular la creación de equipos multidisciplinarios para atender a estos enfermos y pensamos que el internista podría jugar un papel fundamental en la asistencia integral de esta patología.
11. Lyons AR. Clinical outcomes and treatment of hip fractures. Am J Med 1997; 103(2A): 51S-63S.

12. Magaziner J, Simonsick EM, Kashner K, Hebel JR, Kenzora JE. Predictor of functional recovery one year following hospital discharge for hip fracture: a prospective study. J Gerontol 1990; 45: 101-7.

13. Galvard H, Elmstahl S, Elmstahl B, Samuelsson S-M, Robertsson E. Differences in body composition between female geriatric hip fracture patients and healthy controls: body fat is more important as explanatory factor for the fracture than body weight and lean body mass. Age Clin Exp Res 1996; 8: 282-6.

14. Maffulli N, Dougall TW, Brown MTF, Golden MHN. Nutritional differences in patients with proximal femoral fractures. Age and Ageing 1999; 28: 458-62.

15. Hinton RY, Lennox DW, Ebert FR, Jacobsen SJ, Smith GS. Relative rates of the fracture of the hip in the United States. Geographic, sex and age variations. J Bone Joint Surg 1995; 77-A: 695-702.

16. Ray WA, Griffin MR, Schaffner W et al. Psychotropic drugs use and the risk of hip fractures. New Engl J Med 1987; 316: 363-8.

17. Incalzi RA, Capparella O, Gemma A, Camaioni D, Sanguinetti C, Carbonin PU. Predicting in-hospital mortality after hip fracture in elderly patients. J Trauma 1994; 36: 79-82.

18. White BL, Fisher WD, Laurin CA. Rate of mortality for elderly patients after fracture of the hip in the 1980's. J Bone Joint Surg Am 1987; 69: 1335-40.

19. Méndez JM, Girvent R, Arman A, Huguet J, Gordo F, Martí J. Factores pronósticos en la mortalidad y morbilidad de las fracturas del tercio proximal del fémur. Rev Ortop Trauma 1997; 41: 407-10.

20. Sáez-Aldana F, Martínez-Galarreta MV, Martínez-Íñiguez Blasco J. Análisis de las caídas productoras de fractura de cadera en el anciano. Rev Ortop Traumatol 1999; 2: 99-106. 
21. Schürch MA, Rizzoli R, Mermillod B, Vasey H, Michel JP, Bonjour JP. A prospective study on socioeconomic aspects of fracture of the proximal femur. J Bone Min Res 1996; 11: 1935-1942.

22. Michelson JD, Myers A, Jinnah R, Cox Q, Van Natta M. Epidemiology of hip fractures among the elderly: risk factors for fracture type. Clin Orthop Relat Res 1995; 311: 129-35.

23. Bastow MD, Rawlings J, Allison SP. Benefits of supplementary tube feeding after fractured neck of femur: a randomised controled trial. $\mathrm{Br}$ Med J 1983; 287: 1589-92.

24. Patterson BM, Cornell CN, Carbone B, Levine B, Chapman D. Protein deplection and metabolic stress in elderly patients who have a fracture of the hip. J Bone and Joint Surg 1992; 74-A: 251-60.

25. Foster MR, Heppenstall RB, Friedemberg ZB, Hozack WJ. A prospective assessment of nutritional status and complications in patients with fractures of the hip. J Orthop Trauma 1990; 4: 49-57.

26. Nelson KM, Richards EW, Long CL, Martin KR, Geiger JW, Brooks $\mathrm{SW}$, et al. Protein and energy balance following femoral neck fracture in geriatric patients. Metabolism 1995; 44: 59-66.

27. LeBoff MS, Kohlmeier L, Hurwitz S, Franklin J, Wright J Glowacki J. Occult vitamin D deficiency in postmenopausal US women with acute hip fracture. JAMA 1999; 281 (16): 1505-11.

28. Diamond T, Smerdely P, Kormas N, Sekel R, Vu T, Day P. Hip fracture in elderly men: the importance of subclinical vitamin $\mathrm{D}$ deficiency and hipogonadism. Med J Aust 1998; 169 (3): 138-41.

29. Martínez ME, del Campo MT, García JA, Sánchez-Cabezudo MJ, Medina S, García-Cimbrelo E, et al. Concentraciones de vitamina D en pacientes con fractura de cadera en Madrid. Med Clin (Barc) 1996; 106: 41-4.

30. Jallut D, Tappy L, Kohut M, et al. Energy balance in elderly patients after surgery for a femoral neck fracture. JPEN 1990; 14: 563-8.

31. Mullen JO, Mullen NL. Hip fracture mortality: a prospective multifactorial study to predict and minimize death risk. Clin Orthop Relat Res 1992; 280: 214-22.

32. Tkatch L, Rapin CH, Rizzoli R, Slosman D, Nydegger V, Vasey H, et al. Benefits of oral protein supplementation in elderly patients with fracture of the proximal femur. J Am Coll Nutr 1992; 11: 519-25.

33. Delmi M, Rapin CH, Bengoa JM, Delmas PD, Vasey H, Bonjour JP. Dietary supplementation in elderly patients with fractured neck of the femur. Lancet 1990; 335: 1013-6.

34. Benet J, Domínguez A, Sales JM, Orozco R, Salleras L. In-hospital case-fatality of aged patients with hip fracture in Catalonia, Spain. Eur J Epidemiol 1997; 13: 681-6.

35. Sosa Henríquez M, Segarra MC, Limiñana JM, Hernández D, González A, Betancor P. Morbilidad y mortalidad de la fractura osteoporótica de la extremidad proximal del fémur tras un año de seguimiento. Med Clin (Barc) 1993; 101: 481-3.

36. Cree M, Soskolne CL, Belseck E, Hornig J, McElhaney JE, Brant R, et al. Mortality and institutionalization following hip fracture. J Am Geriatr Soc 2000; 48: 283-8.

37. Lu-Yao GL, Baron JA, Barrett JA, Fisher ES. Treatment and survival among elderly americans with hip fractures: a population-based study. Am J Publ Health 1994; 84: 1287-91.

38. Schrøder HM, Erlandsen M. Age and sex as determinants of mortality after hip fracture: 3.895 patients followed for 2.5-18.5 years. J Orthop Trauma 1993; 6: 525-31.

39. Tonetti J, Couturier P, Rémy A, Nicolas L, Merloz P, Franco A. Fracture de 1'extrémité supérieure du fémur après 75 ans: pronostic vital et fonctionnel d'une cohorte de 78 patients suivie 2,5 ans. Rev Chirur Orthop 1997; 83: 636-44.

40. Kuokkanen HOM, Korkala OL. Factors affecting survival of patients with hip fractures. Acta Orthop Belgica 1992; 58: 425-8.

41. Koval KJ, Zuckerman JD. Current concepts review. Functional recovery after fracture on the hip. J Bone Joint Surg 1994; 76 A: 751-8.

42. Jette AM, Harris BA, Cleary PD, Campion EW. Functional recovery after hip fracture. Arch Phys Med Rehabil 1987; 68: 735-40.

43. Koval KJ, Skovron ML, Aharonoff GB, Meadows SB, Zuckerman JD. Ambulatory ability after hip fracture: a prospective study in geriatric patients. Clin Orthop 1995; 310: 150-9.

44. Meadows SE, Zuckerman JD, Sakales SR, Frankel VH. Ambulatory ability after hip fracture: a prospective study in geriatric patients. Orthop Trans 1991; 15: 700 .

45. Martín Peña G, Canalejo Castrillero E. Composición corporal. Valoración del estado nutricional. En: Tratado de nutrición artificial. Celaya Pérez S. Ed. Grupo Aula Médica, Madrid 1998.

46. Christie PM, Hill GL. Effect of intravenous nutrition on nutrition and function in acute attacks of inflamatory bowel disease. Gastroenterology 1990; 99: 730-6.

47. Bonjour JP, Rizzoli R. Inadequate protein intake and osteoporosis : possible involvement of the IGF sistem. Challenges. Mod Med 1995: 399-406.

48. Vellas B, Baumgartner RN, Wayne SJ, Conceicao J, Lafont C, Albarede JL, et al. Relationship between malnutrition and falls in the elderly. Nutrition 1992; 8: 105-8.

49. Constans T, Bacq Y, Bréchot JF, Guilmot JL, Choutet P, Lamisse F. Protein-energy malnutrition in elderly medical patients. J Am Geriatr Soc 1992; 40: 263-8.

50. Manciet G, Galley P, Emeriau JP, Boisnier A, Borede C, Angibault R. Enquête prospective dans un service de médicine interne, a propos de 400 observations. Rev Franç Endocrinol Clin 1983; 24: 225-36.

51. Esteban Pérez M, Fernández-Ballart J, Salas-Salvadó. Estado nutricional de la población anciana en función del régimen de institucionalización. Nutrición Hospitalaria 2000; 15: 105-13.

52. Lumbars M, Driver L, Howland R, Older M, Williams C. Nutritional status and clinical outcome in elderly female surgical orthopedic patients. Clin Nutr 1996; 15: 101-7.

53. Bruun LI, Bosaeus I, Bergstad I, Nygaard K. Prevalence of malnutrition in surgical patients: evaluation of nutritional support and documentation. Clin Nutr 1999; 18: 141-7.

54. Campillo B, Paillaud E, Boires PN, Noel M, Porquet D, Le Parco JC. Serum Levels of insulin-like growth factor-1 in the three months following surgery for a hip frcture in elderly: relationship with nutritional status and inflamatory reaction. Clin Nutr 2000; 19: 349-54.

55. Van-Hoang H, Silverstone FA, Leventer S, Wolf-Klein GP, Foley CJ. The effect of nutritional status on length of stay in elderly hip fracture patients. J Nutr Health Aging 1998; 2: 159-61.

56. Lumbers M, New SA, Gibson S, Murphy MC. Nutritional status in elderly female hip fractured patients: comparison with an age-matched home living group attending day centres. Br J Nutr 2001; 85: 733-40.

57. Bachrach-Lindström M, Unosson M, Ek AC, Arnqvist H. Assessment of nutritional status using biochemical and anthropometric variables in a nutritional intervention study of women with hip fracture. Clin Nutr 2001; 20: 217-23.

58. Fitzsimmons G. Nutritional assessment and the effects of protein supplementation in elderly patients with fractured neck of femur admitted to Middlemore Hospital. NZ Dietetic Training School Annual Report 1997: 10.

59. Paillaud E, Campillo B, Boires PN, Le Parco JC. Évaluation de l'état nutritionnel de 57 malades âgés hospitalisés: influence de la pathologie causale. Rev Méd Interne 2001; 22: 238-44.

60. Fleck A. Acute phase response: implications for nutrition and recovery. Nutrition 1988; 4: 109-17.

61. Joosten E, Vanderelst B, Pelemans W. The effect of different diagnostic criteria on the prevalence of malnutrition in a hospitalized geriatric population. Aging Clin Exp Res 1999; 11: 390-4.

62. Dreblow DM, Anderson CF, Moxness K. Nutritional assessment of orthopedic patients. Mayo Clin Proc 1981; 56: 51-54.

63. Schurch MA, Rizzoli R, Slosman D, Vadas L, Vergnaud P, Bonjour Jp. Protein supplements increase serum insulin-like growth factor-I levels and attenuate proximal femur bone loss in patients with recent hip fracture: a randomized, double-blind, placebo-controlled trial. Ann Intern Med 1998; 128: 801-9.

64. Mora JK. Malnutrition; organic and functional consequences. World J Surg 1999; 23: 530-5.

65. Esposito $\mathrm{S}$. Immune system and surgical site infection. J Chemother 2001; $13 \operatorname{Spec}^{\circ} 1$ (1): 12-6.

66. Puskarich CL, Nelson CL, Nusbickel FR, Stroope HF. The use of two nutritional indicators in identifying long-bone fracture patients who do and do not develop infections. J Orthop Res 1990; 8: 799-803.

67. Lesourd BM. Nutrition and immunity in the elderly: modification of immune responses with nutritional treatment. Am J Clin Nutr 1997; 66 (Supl.): 478-84.

68. Kawakami K, Kadota J, Tida K, Shirai R, Abel K. Reduced immune function and malnutrition in the elderly. J Exp Med 1999; 187: 157-171.

69. Allman RM, Goode PS, Patrick MM, Burst N, Bartolucci AA. Pressure ulcer risk factors among hospitalized patients with activity limitation. JAMA 1995; 274: 1014-5.

70. Russell L. The importance of patients' nutritional status in wound healing. Br J Nurs 2001; 10 (Supl. 6): S42, S44-49.

71. Singer P. Nutritional care to prevent and heal pressure ulcers. Isr Med Assoc J 2002; 4: 713-6.

72. Christensson I, Unosson M, Ek AC. Malnutrition in elderly people newly admitted to a community resident home. J Nutr Health and Aging 1999; 3: 133-9. 
73. Sullivan DH, Nelson CL, Bopp MM, Puskarich-May CL, Walls RC. Nightly enteral nutrition support of elderly hip fracture patients: a phase I trial. J Am Coll Nutr 1998; 17: 155-61.

74. Incalzi RA, Landi F, Cipriani L, Bruno E, Pagano F, Gemma A, et al. Nutritional assessment: a primary component of multidimensional geriatric assessment in the acute care setting. J Am Geriatr Soc 1996; 44 (2): 166-74.

75. Rudman D, Feller AG. Protein-calorie malnutrition in the nursing home. J Am Geriatr Soc 1989; 37: 173-83.

76. Campbell AJ, Spears GFS, Brown JS, et al. Anthropometric measurements as predictors of mortality in a community population aged 70 years and over. Age Aging 1990; 19: 131.

77. Gill TM, Robinson JT, Tinetti ME. Predictors of recovery in activities of daily living among disabled older person living in the community. $\mathbf{J}$ Gen Intern Med 1997; 12: 757-62.

78. Ponzer S, Tidermark J, Brismar K, Soderqvist A, Cederholm T. Nutritional status, insulin-like growth factor-1 and quality of life in elderly women with hip fracture. Clin Nutr 1999; 18: 241-6.

79. Unosson M, Larsson J, Ek AC, Bjurulf B. Effects of dietary supplement on functional condition and clinical outcome measured with a modified Norton scale. Clin Nutr 1992; 11: 134-9.

80. Volkert D, Hubsch S, Oster P, Schlierf G. Nutritional support and functional status in undernourished geriatric patients during hospitalization and 6-month follow-up. Aging 1996; 8: 386-95.

81. Altadill A, Gómez C, Virgós MJ, Díaz B, Cannata JB. Epidemiología de la fractura de cadera en Asturias. Med Clin (Barc) 1995; 105: 281-6.

82. Myers AH, Robinson EG, Van Natta ML, Michelson JD, Collins K, Baker SP. Hip fractures among the elderly: factors associated with inhospital mortality. Am J Epidemiol 1991; 134: 1128-37.

83. Poor G, Atkinson EJ, O'Fallon WM, Melton LJ. Determinants of reduced survival following hip fractures in men. Clin Orthop Relat Res 1995; 319: 260-5.

84. Hoerer D, Volpin G, Stein H. Results of early and delayed surgical fixation of hip fractures in the elderly: a comparative retrospective study. Bull Hosp Joint Dis 1993; 53: 29-33.

85. Davis TRC, Sher JL, Porter BB, Checketts RG. The timing of surgery of intertrochanteric femoral fractures. Injury 1988; 19: 244.

86. Eiskjaer S, Ostgard SE. Risk factors influencing mortality after bipolar hemiarthroplasty in the treatment of fracture of the femoral neck. Clin Orthop Relat Res 1989; 270: 295-300.

87. Dolk T. Influence of treatment factors on the outcome after hip fractures. Upsala J Med Sci 1989; 94: 209-21.

88. Kenzora JE, McCarthy RE, Lowell JD, Sledge CB. Hip fracture mortality, relation to age, treatment, preoperative illness, time of surgery and complications. Clin Orthop 1984; 186: 45-56.

89. Zohman GL and Lieberman JR . Perioperative aspects of hip fractures. Guidelines for intervention that will impact prevalence and outcome. Am J Orthop 1995; 24: 666-71.

90. Parker MJ, Palmer CR. A new mobility score for predicting mortality after hip fracture. J Bone Jt Surg (Br) 1993; 75B: 797-8.
91. Ensberg MD, Paletta MJ, Galecki AT, Dacko CL, Fries BE. Identifying elderly patients for early discharge after hospitalisation for hip fracture. J Gerontol 1993; 48: 187-95.

92. Duke RG, Keating JL. An investigation of factors predictive of independence in transfer and ambulation after hip fracture. Arch Phys Med Rehabil 2002; 83: 158-64.

93. Koot VC, Peeters PH, de Jong JR, Clevers GJ, van der Werken C. Functional results after treatment of hip fracture: a multicentre prospective study in 215 patients. Eur J Surg 2000; 166: 480-5.

94. Hannan EL, Magaziner J, Wang JJ, Eastwood EA, Silberzweig SB, Gilbert $\mathrm{M}$ et al. Mortality and locomotion 6 months after hospitalization for hip fracture: risk factors and risk-adjusted hospital outcomes. JAMA 2001; 285: 2736-42.

95. Kitamura S, Hasegawa Y, Suzuki S, Sasaki R, Iwatw H, Wingstrand H et al. Functional outcomes after hip fracture in Japan. Clin Orthop 1998; 348: 29-36.

96. Koval KJ, Skovron ML, Polatsch D, Aharonoff GB, Zuckerman JD. Dependency after hip fracture in geriatric patients: a study of predictive factors. J Orthop Trauma 1996; 10: 531-5.

97. Koval KJ, Skovron ML, Aharonoff GB, Zuckerman JD. Predictors of functional recovery after hip fracture in the elderly. Clin Orthop 1998; 348: 22-8.

98. Young Y, Brant L, German P, Kenzora J, Magaziner J. A longitudinal examination of functional recovery among older people with subcapital hip fractures. J Am Gariatr Soc 1997; 45: 288-94.

99. Michel JP, Klopfenstein C, Hoffmeyer P, Stern R, Grab B. Hip fracture surgery: is the preoperative American Society of Anesthesiologist (ASA) score a predictor of functional outcomes? Aging Clin Exp Res 2002; 14: 389-94.

100. Gustilo RB, Kyle RF, Tepleman D. Fracturas y luxaciones. Volumen II. Editorial Panamericana 1995; Capítulo 21. p. 800.

101. Marottoli RA, Berkman LF, Cooney LM. Decline in physical function following hip fracture. J Am Gariatr Soc 1992; 40: 861-6.

102. Fink A, Hays RD, Moore AA, Beck JC. Alcohol-related problems in older persons. Determinants, consequences and screening. Arch Intern Med 1996; 156: 1150-6.

103. Tanaka J, Tokimura F, Seki N. Outcomes after hip fractures surgery in patients aged $>$ or $=90$ years. Orthopedics 2003; 26: 55-8.

104. Goldstein FC, Strasser DC, Woodard JL, Roberts VJ. Functional outcome of cognitively impaired hip fracture patients on a geriatric rehabilitation unit. J Am Geriatr Soc 1997: 45: 35-42.

105. Youm T, Aharonoff GB, Zuckerman JD, Koval KJ. Effect of previous cerebrovascular accident on outcomes after hip fracture. J Orthop Trauma 2000; 14: 329-34.

106. Marcantonio ER, Flaker JM, Michaels M, Resnick NM. Delirium is independently associated with poor functional recovery after hip fracture. J Am Geriatr Soc 2000: 48: 618-24.

107. Morrison RS, Chassin MR, Siu AL. The medical consultant's role in caring for patients with hip fracture. An Intern Med 1998; 128: 101020. 